\title{
P25 PUTTING PATIENTS IN CONTROL
}

Carole Dickinson, Julie Tydeman Royal Derby Hospital, Derby, UK

10.1136/bmjspcare-2011-000105.25

Background The Nightingale Macmillan Unit is a purpose built 20 bed specialist palliative care unit within an acute hospital. Staff identified that patients with impaired upper limb function had difficulty or were unable to summon assistance of staff on the ward. In addition, some patients who had environmental controls at home reported to feel extremely vulnerable and dependant during respite admissions, as there were no such facilities at the unit. During respite admissions we wish to maintain the patients function and condition to ensure that they can return home with the pre-existing care package and equipment.

Methods Having identified the problem, discussions were had with patients from the respite list as to what equipment they used at home, and what was most important to them. A number of different staff groups then worked together to obtain a range of non-standard nurse call switches and an environmental control system to facilitate control of electrical appliances. Results Patients with impaired upper limb function can now use the nurse call system to summon assistance when they need it. Patients now also have control over lighting, TV, radio and a fan.

Discussion These improvements have enabled patients with impaired upper limb function to feel more empowered during their respite admissions. The non-standard nurse call switches have allowed patients to summon assistance when they need it. Therefore patient's privacy and dignity are greater and demands on nursing staff time are reduced. "When I wake up in the night, I can now turn on the light instead of being stuck in the dark" Gordon (patient). It has been both a positive and rewarding experience for staff to be able to use technology to promote patient independence and quality of life. 